\title{
Optimal blood pressure target and measurement in patients with chronic kidney disease
}

\author{
Chang Seong Kim, Hong Sang Choi, Eun Hui Bae, Soo Wan Kim, and Seong Kwon Ma
}

Department of Internal Medicine, Chonnam National University Medical School, Gwangju, Korea

Received: March 18, 2019

Accepted: May 19, 2019

\section{Correspondence to}

Seong Kwon Ma, M.D.

Department of Internal Med-

icine, Chonnam National

University Medical School, 42

Jebong-ro, Dong-gu, Gwangju

61469 , Korea

Tel: +82-62-220-6579

Fax: +82-62-225-8578

E-mail:drmsk@jnu.ac.kr

https://orcid.org/0000-0002-

5758-8189
The prevalence rates of hypertension and chronic kidney disease (CKD) are increasing with the aging of the population. Hypertension and CKD are closely related, and hypertension with accompanying CKD is difficult to control. This difficulty controlling blood pressure (BP) can be explained by changes in diurnal variation in BP, such as non-dipping and reverse dipping patterns, increased pulse pressure, and BP variability in CKD patients resulting in a high frequency of nocturnal hypertension or masked hypertension. CKD patients with uncontrolled or nocturnal hypertension are at increased risk for cardiovascular disease, progression of CKD, and all-cause death. Recent studies have shown that intensive reduction of systolic BP below $120 \mathrm{mmHg}$ is seems to favor in CKD patients regardless of the presence or absence of diabetes. As BP control is difficult in patients with CKD, appropriate measurement of BP is important. Automated BP monitoring could reduce the so-called "white coat effect" (spike in BP) that may be triggered by measurement in a clinical setting. Moreover, out-of-office BP monitoring at home or ambulatory BP monitoring for 24 hours may provide critical information regarding diurnal BP variability and nocturnal BP in patients with CKD.

Keywords: Hypertension; Blood pressure monitoring; Kidney diseases

\section{INTRODUCTION}

According to data from the World Health Organization, hypertension is estimated to affect up to $40 \%$ of adults worldwide, and the prevalence is continuously increasing. According to the Korean Hypertension Fact Sheet 2018, the age-standardized mean blood pressure (BP) levels have remained almost unchanged over the last 10 years. Nevertheless, the prevalence of hypertension is $29 \%$ in the adult Korean population aged 30 years or older. Furthermore, with continued aging of the population, the estimated size of the population with hypertension is over over 11 million in Korea. It is thought to be impossible to control BP in over half of these cases, representing 5.9 million individuals [1]. Chronic kidney disease (CKD) is strongly correlated with hypertension. In a previous study, a higher CKD stage was found in patients with hypertension than in those with normal $\mathrm{BP}$, and time-varying systolic BP was associated with an increase in the risk of developing CKD, with the risk increasing above $120 \mathrm{mmHg}$ [2]. The Chronic Renal Insufficiency Cohort (CRIC) study reported that the prevalence of hypertension was $86 \%$ in patients with CKD, whereas only $46 \%$ of patients had $\mathrm{BP}<130 / 80 \mathrm{mmHg}$, which was controlled according to the recent 2017 American College of Cardiology/American Heart Association (ACC/AHA) hypertension guidelines [3]. In addition, patients with CKD in a large community-based study were reported to be at greater risk for hypertension-related adverse outcomes, including risk for death, 
cardiovascular events, and hospitalization [4]. In another study, CKD patients with hypertensive nephropathy, such as diabetic nephropathy, were at 3.3-fold higher risk for cardiovascular events and death than those with primary renal disease [5]. Hypertension is common in patients with CKD, and uncontrolled hypertension is associated with adverse clinical outcomes.

Therefore, strict BP control is needed to reduce cardiovascular events and death in patients with CKD. The aim of this review was to identify the acceptable BP target with regard to safety and benefits, and to determine the altered circadian BP variability and usefulness of out-of-office BP monitoring for proper BP measurement in CKD patients.

\section{LOW BP TARGET IN CKD PATIENTS: SAFETY AND BENEFITS}

While the risk for cardiovascular disease increases with increasing BP, and lowering BP reduces this risk in patients with hypertension, the optimal BP target to prevent hypotension-associated adverse events has not been elucidated [6]. Three randomized controlled trials (the Modification of Diet in Renal Disease [MDRD] study, the African American Study of Kidney Disease and Hypertension [AASK], and the Ramipril Efficacy In Nephropathy [REIN]-2 trials) indicated beneficial effects of low BP targets in reducing cardiovascular events, mortality, and slowing the progression of CKD to end-stage renal disease in patients with CKD [7-9]. However, a recent randomized controlled trial in patients with non-diabetic CKD, i.e., the Systolic Blood Pressure Intervention Trial (SPRINT), raised questions regarding whether intensive systolic BP control reduces risk for cardiovascular disease and all-cause death [10]. The SPRINT study reported a $25 \%$ risk reduction in the composite of cardiovascular disease and a $27 \%$ decrease in death from any cause in an intensive treatment group (systolic $\mathrm{BP}<120 \mathrm{mmHg}$ ) compared to a standard treatment group (systolic $\mathrm{BP}<140 \mathrm{mmHg}$ ) [10]. Subgroup analyses indicated no differences in primary outcome between the intensive treatment group and the standard treatment group among patients with CKD. $A \geq 30 \%$ reduction in estimated glomerular filtration rate (eGFR) to $<60 \mathrm{~mL} / \mathrm{min} / 1.73 \mathrm{~m}^{2}$ in a sub- population without CKD was significantly higher in the intensive treatment group than in the standard treatment group [10]. Moreover, although most participants showed complete recovery of kidney function, intensive BP lowering resulted in a 1.6-fold increase in episodes of acute kidney injury in all subjects [11]. Therefore, there has been some debate as to whether intensive BP lowering may be harmful in patients with CKD. However, in a recent study of 2,646 SPRINT participants with non-diabetic CKD, the risk for all-cause death was significantly lower with a target systolic $\mathrm{BP}<120$ $\mathrm{mmHg}$ compared to $<140 \mathrm{mmHg}$ [12]. In addition, according to a study that employed urine biomarkers, the eGFR decline during the first 6 months in the intensive arm of SPRINT predominantly reflected hemodynamic changes rather than intrinsic damage to kidney tubule cells [13]. Similar to the results of a previous urine biomarker study, observations in participants with diabetic CKD in the Action to Control Cardiovascular Disease in Diabetes Blood Pressure (ACCORD-BP) trial also showed that intensive BP control was associated with reductions in eGFR but not with increases in injury marker levels, suggesting that they reflect hemodynamic changes $[13,14]$. Thus, on balance, the best strategy seems to be intensive systolic BP lowering in the CKD population, regardless of the presence or absence of diabetes [15]. The 2012 Kidney Disease Improving Global Outcomes (KDIGO) guidelines suggest a BP target of < 130/80 mmHg in CKD patients with proteinuria, whereas the $2017 \mathrm{ACC} / \mathrm{AHA}$ guideline suggests adjustment to $<130 / 80 \mathrm{mmHg}$ even without proteinuria $[16,17]$.

\section{DIURNAL VARIATION IN BP IN CKD PATIENTS}

When BP targets are determined, precise BP should be measured to diagnose hypertension and control BP target levels. However, the altered circadian BP rhythm in patients with CKD is an obstacle to the diagnosis and control of BP, in contrast to healthy individuals [18,19]. Indeed, healthy individuals have a dipping pattern in which BP falls by $10 \%$ to $20 \%$ during sleep, whereas the dipping pattern is blunted in individuals with CKD $[20,21]$. A previous study showed a significantly increased prevalence of non-dipping (blunted nocturnal BP decline) or riser patterns (greater increase in BP 
during sleep than when awake) in hypertensive patients with CKD [20]. The AASK reported that $80 \%$ of participants had a non-dipping or reverse dipping pattern [22]. In addition, the prevalence of the non-dipping pattern was higher among patients with CKD than in those without CKD (60.6\% vs. $43.2 \%$, respectively) [23]. Nocturnal hypertension can be diagnosed when office/ clinical BP is within the normal range during the daytime but increases at night. Thus, masked hypertension is associated with nocturnal hypertension, particularly the riser pattern that results in a missed diagnosis of hypertension and undertreatment of hypertensive patients with CKD. Remarkably, among CKD patients with normotension based on clinical BP, the prevalence of masked hypertension has been reported to be as high as $40 \%$ to $70 \%$ [24]. Previous studies have shown that low eGFR and proteinuria are associated with nighttime and masked hypertension [25-27]. The mechanisms underlying the altered diurnal BP variability are incompletely understood. Nevertheless, increased sodium retention, fluid overload, activation of the renin-angiotensin system and endothelin, altered sympathetic nervous system activity, endothelial dysfunction, oxidative stress, and arterial stiffness have been proposed to explain the pathophysiology of altered BP variability with decreased renal function, such as nocturnal hypertension, a non-dipping or riser pattern during sleep, increased pulse pressure, and exaggerated morning BP surges [28-30]. Eventually, these altered circadian BP rhythms cause target organ damage and eventually have detrimental effects on the progression of cardiovascular disease and kidney disease. Compared to individuals with controlled clinical BP or white coat hypertension, the risks of cardiovascular and kidney disease progression are higher in those with elevated nocturnal BP, masked hypertension, or sustained hypertension accompanying CKD and in those undergoing hemodialysis $[20,22,23]$. Therefore, precise and optimal BP measurement is needed to determine masked hypertension and nocturnal hypertension because these are common characteristics of patients with CKD.

\section{OPTIMAL BP MEASUREMENT IN CKD PATIENTS}

There are two methods of measuring BP: office-based and out-of-office-based measurements (Fig. 1). The diagnosis and management of hypertension are still mainly based on office-measured BP. However, traditional measurement using an aneroid sphygmomanometer is limited by a lack of medical staff, differences between measurer, an inability to assess circadian variation, and the "white coat effect" defined as transient increases in BP observed in some patients when confronted with medical staff leading to overdiagnosis and overtreatment of hypertension [31].

On the other hand, automated office BP measurement (AOBPM), which is widely available in offices and provides multiple consecutive $\mathrm{BP}$ readings with the patient resting alone, has been shown to decrease the white coat effect compared to single manual BP measurements. Indeed, the ACCORD-BP and SPRINT trials measured BP using AOBPM, which yielded approximately 5 to $10 \mathrm{mmHg}$ lower values than typical office systolic BP readings [31,32]. Recent studies have shown that AOBPM is a valuable method for predicting cardiovascular events [33,34].

The next method of BP measurement is home blood pressure monitoring (HBPM). The wide availability of automated electronic devices could increase the use of HBPM. Hypertensive patients with CKD who use HBPM are more likely to be at or below the target BP level [35]. A previous meta-analysis of 18 randomized controlled clinical trials showed that HBPM results in better BP control and greater achievement of BP targets than usual BP monitoring in the hypertensive population [36]. This can be explained by the fact that the direct feedback of one's own BP level may improve motivation in self-care as well as increase adherence to antihypertensive medications [35]. In predicting clinical outcomes, a prospective cohort study performed in 150 patients undergoing chronic hemodialysis showed that HBPM was associated with a $40 \%$ to $63 \%$ higher rate of all-cause or cardiovascular mortality for a 1-standard deviation (SD) increment in diastolic BP [37]. Consequently, HBPM can predict associations more strongly with target organ damage and progression of CKD compared to routine office BP measurements $[38,39]$. All measurements at home should be made at the same times of day each day and BP measurements should be averaged after 2 to 4 weeks to assess the effects of antihypertensive treatment [38]. Patients must verify that 

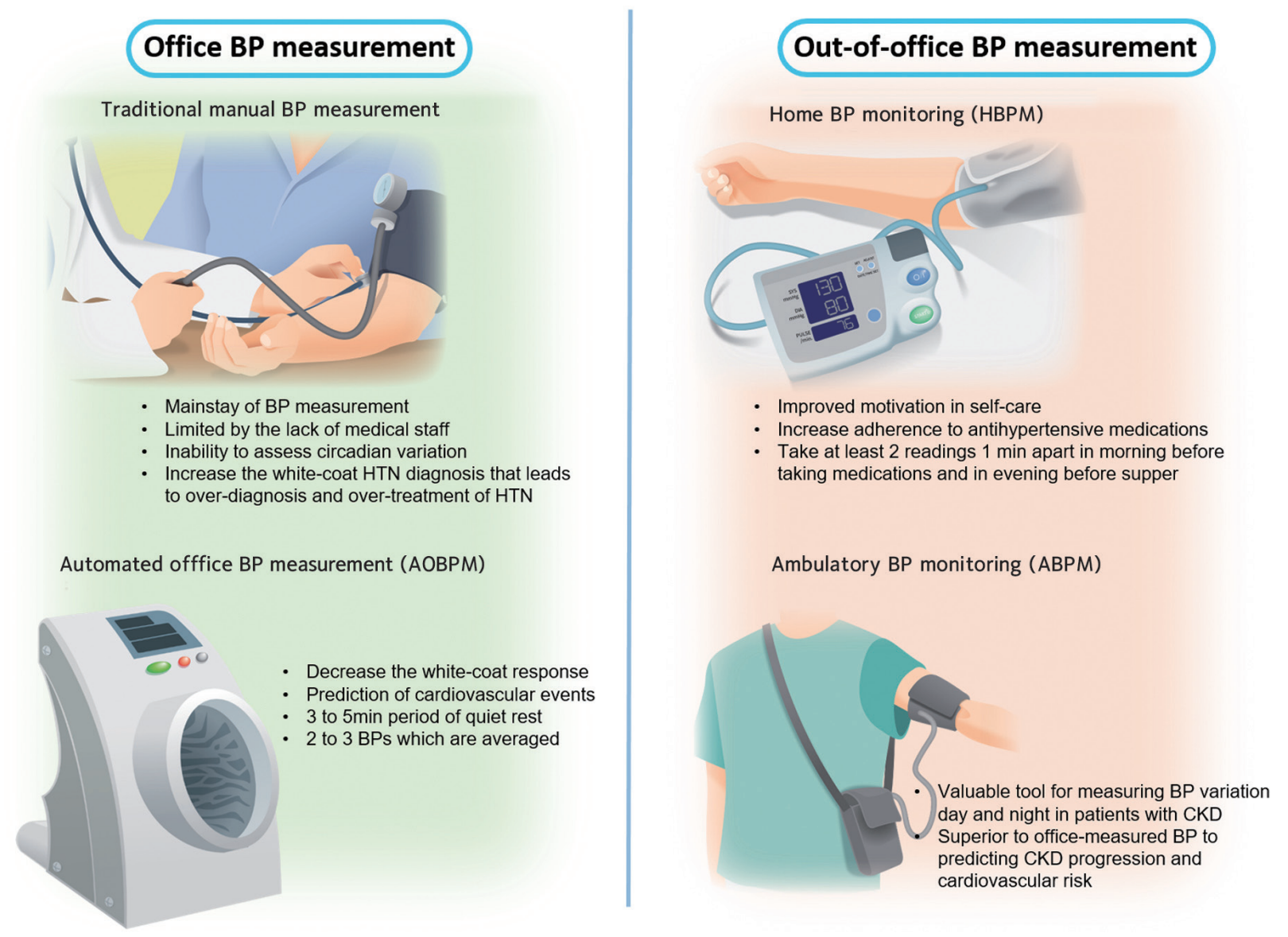

Figure 1. Methods of measuring blood pressure: office-based and out-of-office-based blood pressure (BP) measurements. CKD, chronic kidney disease; HTN, hypertension.

differences between the left and right arms are insignificant. If differences are significant, the physician must instruct the patient to measure BP in the arm with higher readings. In addition, BP should be checked in the morning before taking medications and in the evening before supper $[16,38]$. Furthermore, regardless of typical manual office BP measurement, AOBPM and HBPM must be measured accurately. To reduce intrapatient variability, BP must be measured after 5 minutes of rest, two to three readings should be taken 1 to 2 minutes apart, and BP measurements should be averaged. In addition, a cuff with a bladder encircling $80 \%$ of the arm must be used. The cuff should be placed such that the middle is on the patient's bare upper arm at the level of the right atrium (the midpoint of the sternum) [40,41]. Out-of-office measurements are recommended to at least confirm the diagnosis of hy- pertension and for initiation of antihypertensive medication, in conjunction with telehealth counseling or clinical interventions according to the 2017 ACC/AHA guidelines [16].

As mentioned above, hypertensive patients with CKD exhibit altered diurnal variation in BP, which may include a non-dipping pattern, nocturnal hypertension, sustained hypertension, a morning surge, high $\mathrm{BP}$ variability, and increased pulse pressure. BP must be measured from morning to night, regardless of whether in an office or out-of-office setting. Thus, ambulatory blood pressure monitoring (ABPM) could be helpful for detecting changes in circadian BP variation in patients with CKD, because it measures BP every half hour during the day and every hour during the night over 24 hours [28]. A study conducted on a general Japanese population showed that nighttime BP is a 
better predictor of CKD development than daytime BP, suggesting that $A B P M$ is useful for evaluating the risk of progression to CKD [42]. Therefore, ABPM may be a more valuable tool for measuring variation between daytime and nighttime BP to identify patients at risk for cardiovascular complications than office-based BP measurements or even HBPM $[43,44]$.

\section{CONCLUSIONS}

Hypertension is associated with increased risk for cardiovascular events and death in CKD patients. Recent guidelines recommend a lower BP target in CKD to reduce cardiovascular and all-cause death. However, nocturnal non-dipping, masked hypertension, morning surge, increased pulse pressure, and resistant hypertension are common features of altered diurnal variation in BP in CKD patients. Out-of-office BP measurement, including $\mathrm{HBPM}$ and $\mathrm{ABPM}$, may improve the assessment of overall BP control in patients with CKD compared to office-based BP measurements alone. Although ABPM for 24 hours provides critical information on circadian BP variability and nocturnal BP, if it is difficult to access, HBPM is also recommended and could be applied in patients well informed on how to measure BP accurately.

\section{Conflict of interest}

No potential conflict of interest relevant to this article was reported.

\section{Acknowledgments}

This research was supported by the Bio \& Medical Development Program through the NRF of Korea funded by the Ministry of Science and ICT (MSIT) (NRF-2017M3A9E8023018), and by a grant (CRI 180161, BCRI18025) Chonnam National University Hospital Biomedical Research Institute.

\section{REFERENCES}

1. Korean Society Hypertension (KSH); Hypertension Epidemiology Research Working Group, Kim HC, Cho MC. Korea hypertension fact sheet 2018. Clin Hypertens 2018;24:13.
2. Hanratty R, Chonchol M, Havranek EP, et al. Relationship between blood pressure and incident chronic kidney disease in hypertensive patients. Clin J Am Soc Nephrol 2011;6:2605-2611.

3. Muntner P, Anderson A, Charleston J, et al. Hypertension awareness, treatment, and control in adults with CKD: results from the Chronic Renal Insufficiency Cohort (CRIC) Study. Am J Kidney Dis 2010;55:441-451.

4. Go AS, Chertow GM, Fan D, McCulloch CE, Hsu CY. Chronic kidney disease and the risks of death, cardiovascular events, and hospitalization. N Engl J Med 2004;351:1296-1305.

5. Nakayama M, Sato T, Miyazaki M, et al. Increased risk of cardiovascular events and mortality among non-diabetic chronic kidney disease patients with hypertensive nephropathy: the Gonryo study. Hypertens Res 2011;34:11061110 .

6. Touyz RM. Lower is better in hypertension, but how low should blood pressure be targeted? J Am Soc Hypertens 2016;10:621-622.

7. Klahr S, Levey AS, Beck GJ, et al. The effects of dietary protein restriction and blood-pressure control on the progression of chronic renal disease. Modification of Diet in Renal Disease Study Group. N Engl J Med 1994;330:877884 .

8. Wright JT Jr, Bakris G, Greene T, et al. Effect of blood pressure lowering and antihypertensive drug class on progression of hypertensive kidney disease: results from the AASK trial. JAMA 2002;288:2421-2431.

9. Ruggenenti P, Perna A, Loriga G, et al. Blood-pressure control for renoprotection in patients with non-diabetic chronic renal disease (REIN-2): multicentre, randomised controlled trial. Lancet 2005;365:939-946.

10. SPRINT Research Group, Wright JT Jr, Williamson JD, et al. A randomized trial of intensive versus standard blood-pressure control. N Engl J Med 2015;373:2103-2116.

11. Rocco MV, Sink KM, Lovato LC, et al. Effects of intensive blood pressure treatment on acute kidney injury events in the Systolic Blood Pressure Intervention Trial (SPRINT). Am J Kidney Dis 2018;71:352-361.

12. Cheung AK, Rahman M, Reboussin DM, et al. Effects of intensive BP control in CKD. J Am Soc Nephrol 2017;28:28122823.

13. Malhotra R, Craven T, Ambrosius WT, et al. Effects of intensive blood pressure lowering on kidney tubule injury in CKD: a longitudinal subgroup analysis in SPRINT. Am 
J Kidney Dis 2019;73:21-30.

14. Nadkarni GN, Chauhan K, Rao V, et al. Effect of intensive blood pressure lowering on kidney tubule injury: findings from the ACCORD trial study participants. Am J Kidney Dis 2019;73:31-38.

15. Walther CP, Shah M, Navaneethan SD. Estimated GFR decline and tubular injury biomarkers with intensive blood pressure control. Am J Kidney Dis 2019;73:4-7.

16. Whelton PK, Carey RM, Aronow WS, et al. 2017 ACC/AHA/ AAPA/ABC/ACPM/AGS/APhA/ASH/ASPC/NMA/PCNA guideline for the prevention, detection, evaluation, and management of high blood pressure in adults: a report of the American College of Cardiology/American Heart Association Task Force on Clinical Practice Guidelines. Hypertension 2018;71:e13-e115.

17. Taler SJ, Agarwal R, Bakris GL, et al. KDOQI US commentary on the 2012 KDIGO clinical practice guideline for management of blood pressure in CKD. Am J Kidney Dis 2013;62:201-213.

18. Kalaitzidis RG, Elisaf MS. Treatment of hypertension in chronic kidney disease. Curr Hypertens Rep 2018;20:64.

19. Goto N, Uchida K, Morozumi K, et al. Circadian blood pressure rhythm is disturbed by nephrectomy. Hypertens Res 2005;28:301-306.

20. Mojon A, Ayala DE, Pineiro L, et al. Comparison of ambulatory blood pressure parameters of hypertensive patients with and without chronic kidney disease. Chronobiol Int 2013;30:145-158.

21. Kario K, Tomitani N, Matsumoto Y, et al. Research and development of information and communication technology-based home blood pressure monitoring from morning to nocturnal hypertension. Ann Glob Health 2016;82:254-273.

22. Pogue V, Rahman M, Lipkowitz M, et al. Disparate estimates of hypertension control from ambulatory and clinic blood pressure measurements in hypertensive kidney disease. Hypertension 2009;53:20-27.

23. Agarwal R, Andersen MJ. Correlates of systolic hypertension in patients with chronic kidney disease. Hypertension 2005;46:514-520.

24. Bangash F, Agarwal R. Masked hypertension and whitecoat hypertension in chronic kidney disease: a meta-analysis. Clin J Am Soc Nephrol 2009;4:656-664.

25. Drawz PE, Alper AB, Anderson AH, et al. Masked hypertension and elevated nighttime blood pressure in CKD: prevalence and association with target organ damage.
Clin J Am Soc Nephrol 2016;11:642-652.

26. Svenningsen P, Friis UG, Versland JB, et al. Mechanisms of renal $\mathrm{NaCl}$ retention in proteinuric disease. Acta Physiol (Oxf) 2013;207:536-545.

27. Thomas G, Drawz PE. BP measurement techniques: what they mean for patients with kidney disease. Clin J Am Soc Nephrol 2018;13:1124-1131.

28. Velasquez MT, Beddhu S, Nobakht E, et al. Ambulatory blood pressure in chronic kidney disease: ready for prime time? Kidney Int Rep 2016;1:94-104.

29. Fukuda M, Munemura M, Usami T, et al. Nocturnal blood pressure is elevated with natriuresis and proteinuria as renal function deteriorates in nephropathy. Kidney Int 2004;65:621-625.

30. Ohashi N, Isobe S, Ishigaki S, Yasuda H. Circadian rhythm of blood pressure and the renin-angiotensin system in the kidney. Hypertens Res 2017;40:413-422.

31. Drawz PE, Ix JH. BP measurement in clinical practice: time to SPRINT to guideline-recommended protocols. J Am Soc Nephrol 2018;29:383-388.

32. Bakris GL. The implications of blood pressure measurement methods on treatment targets for blood pressure. Circulation 2016;134:904-905.

33. Andreadis EA, Papademetriou V, Geladari CV, Kolyvas GN, Angelopoulos ET, Aronis KN. Home, automated office, and conventional office blood pressure as predictors of cardiovascular risk. J Am Soc Hypertens 2017;11:165-170.

34. Stergiou GS, Tzamouranis D, Protogerou A, Nasothimiou E, Kapralos C. Validation of the Microlife Watch BP Office professional device for office blood pressure measurement according to the International protocol. Blood Press Monit 2008;13:299-303.

35. Townsend RR, Taler SJ. Management of hypertension in chronic kidney disease. Nat Rev Nephrol 2015;11:555-563.

36. Cappuccio FP, Kerry SM, Forbes L, Donald A. Blood pressure control by home monitoring: meta-analysis of randomised trials. BMJ 2004;329:145.

37. Alborzi P, Patel N, Agarwal R. Home blood pressures are of greater prognostic value than hemodialysis unit recordings. Clin J Am Soc Nephrol 2007;2:1228-1234.

38. Pickering TG, Miller NH, Ogedegbe G, et al. Call to action on use and reimbursement for home blood pressure monitoring: executive summary: a joint scientific statement from the American Heart Association, American Society Of Hypertension, and Preventive Cardiovascular Nurses Association. Hypertension 2008;52:1-9. 
39. Niiranen TJ, Hanninen MR, Johansson J, Reunanen A, Jula AM. Home-measured blood pressure is a stronger predictor of cardiovascular risk than office blood pressure: the Finn-Home study. Hypertension 2010;55:13461351.

40. de Boer IH, Bangalore S, Benetos A, et al. Diabetes and hypertension: a position statement by the American Diabetes Association. Diabetes Care 2017;40:1273-1284.

41. Powers BJ, Olsen MK, Smith VA, Woolson RF, Bosworth $\mathrm{HB}$, Oddone EZ. Measuring blood pressure for decision making and quality reporting: where and how many mea- sures? Ann Intern Med 2011;154:781-788.

42. Kanno A, Kikuya M, Asayama K, et al. Night-time blood pressure is associated with the development of chronic kidney disease in a general population: the Ohasama Study. J Hypertens 2013;31:2410-2417.

43. Parati G, Ochoa JE, Salvi P, Lombardi C, Bilo G. Prognostic value of blood pressure variability and average blood pressure levels in patients with hypertension and diabetes. Diabetes Care 2013;36:S312-S324.

44. Pickering TG, Shimbo D, Haas D. Ambulatory blood-pressure monitoring. N Engl J Med 2006;354:2368-2374. 